Pasado y Memoria. Revista de Historia Contemporánea,

21, 2020, pp. 179-201

e-ISSN: 2386-4745 | ISSN: 1579-3311

\title{
1968: la contestación estudiantil en Italia y el PCI ${ }^{1}$
}

\author{
1968: the student revolt in Italy and the ICP
}

\author{
Andrea Donofrio \\ Universidad Complutense de Madrid \\ adonofri@ucm.es \\ https://orcid.org/0000-0003-4921-060X
}

Recibido: 12-1-2020

Aceptado: 11-3-2020

Cómo citar este artículo / Citation: DONOFRIO, Andrea (2020). 1968: la contestación estudiantil en Italia y el PCI. Pasado y Memoria. Revista de Historia Contemporánea, 21, pp. 179-201, https://doi.org/10.14198/PASADO2020.21.07

\section{Resumen}

En Italia, el movimiento estudiantil comenzó a adquirir dimensiones de masa ya a finales de 1967. Paulatinamente, despertó el interés político y mediático nacional siendo los sucesos acaecidos en Valle Giulia, Roma, en marzo 1968, el momento de máxima repercusión. Este artículo se propone investigar una serie de cuestiones interdependientes, con el objetivo principal de poner de manifiesto la importancia que tuvo 1968 en el devenir del Partido Comunista italiano (PCI). El punto de partida es la constatación de la existencia de un 'mayo italiano' anterior al francés, que sin duda fue menos popular, pero que contó con sus propias características y peculiaridades. El análisis crítico hace hincapié en la influencia y la repercusión que tuvo el mayo francés en la movilización italiana y, sobre todo, en el debate interno y la reacción del PCI ante estos dos fenómenos.

Palabras clave: 1968; Estudiantes; Partido Comunista italiano; Mayo 1968; Francia; Movimiento estudiantil; Luigi Longo.

1. Este artículo es resultado del Proyecto de Investigación «Diccionario de símbolos políticos y sociales: claves iconográficas, lugares de memoria e hitos simbólicos en el imaginario español del siglo XX» (Ref. HAR2016-77416-P). 


\begin{abstract}
The students' movement began to take on mass dimensions in Italy, already at the end of 1967. It gradually awakened national political and media interest, the events at Valle Giulia in Rome in March 1968 representing its maximum impact moment. This article aims to investigate a series of interdependent issues, with the main objective of highlighting the importance that the year 1968 had for the evolution of the Italian Communist Party (PCI). The starting point is the ascertainment of the existence of an 'Italian May' previous to the French one. While being certainly less popular, it had its own characteristics and peculiarities. Critical analysis places the emphasis on the influence and the impact of the French May on the Italian mobilization and, above all, on the internal debate and reaction of the PCI to these two phenomena.
\end{abstract}

Keywords: 1968; Students; Italian Communist Party; May 68; France; Student movement; Luigi Longo.

\title{
Introducción
}

En Italia, el movimiento de contestación estudiantil nació en los años anteriores a 1968 y se prolongó en los siguientes. Al igual que en otras partes del mundo, los ecos del 68 continuaron en los setenta y sobre todo provocaron una serie de importantes consecuencias políticas, aunque con efectos limitados.

Las protestas de estos años deben enmarcarse en un escenario de crisis global. Una crisis de mitos y de los modelos político-económicos, pero también de valores. El escenario político estaba cambiando ante la aparición de nuevos movimientos, portadores de nuevas reivindicaciones, «nuevas entidades capaces de ejercer una notable fuerza de atracción» (Marzillo, 2012: 271)².

El presente artículo se propone investigar una serie de temas interdependientes, con el objetivo principal de poner de manifiesto la importancia que tuvo 1968 en el equilibrio interno del Partido Comunista italiano (PCI). El punto de partida es la constatación de la existencia de un «68» italiano anterior al francés, que, aunque fue menos popular, contó con sus propias características y peculiaridades. Al mismo tiempo, a través de un análisis crítico de los escritos de los protagonistas políticos de la época, la prensa coetánea y los documentos oficiales del PCI se pondrá el acento sobre la influencia y repercusión que tuvo el mayo francés en la movilización italiana y, sobre todo, en la reacción del PCI ante estos dos fenómenos. Aunque el tema haya sido ya tratado con anterioridad por otros autores, el artículo pretende ofrecer un enfoque inédito y complementario, centrándose sobre todo en la capacidad del PCI de reflexionar sobre el valor político del movimiento estudiantil. De manera concisa se analiza

2. Las referencias en italiano han sido traducidas por el autor del artículo. 
la evolución de la actitud del partido de la incomprensión del alcance de la protesta a la asunción de un estrategia de «atención» y de «dialogo» con el movimiento estudiantil. De enemigo a interlocutor válido, con el objetivo de reconducir el movimiento por los cauces partidistas.

La aparición del movimiento estudiantil representa uno de los momentos más intensos en la dialéctica política interna del PCI durante la secretaría de Luigi Longo. El tema resulta de gran interés ya que a partir de esta fecha-también por la convergencia de otros acontecimientos mundiales de gran calado-el PCI emprendió un intenso debate interno entre los partidarios de una renovación estratégica del partido, acorde a los cambios sociales e internacionales, y aquellos que apostaban por un repliegue identitario en aras de una defensa categórica del socialismo soviético. 1968 con sus dos Primaveras (la de París y la de Praga) marcó el porvenir del PCI en la década de los setenta y su postura eurocomunista. Representó una fecha clave y antecedente a la necesidad de modernización del movimiento comunista italiano, intentando alejarlo de los «reductos dogmáticos» del pasado para dotarlo de un pragmatismo realista más ajustado a la estructura político-social del momento.

\section{7-1968: el despertar juvenil}

En Italia, el movimiento estudiantil empezó a configurarse como organización de masas ya a finales de 1967 tras la ocupación de la Universidad de Trento (sede de la Facultad de Sociología), de la Católica de Milán y del Palacio Campana en Turín. A partir de enero de 1968, con la aproximación del debate parlamentario sobre la nueva ley universitaria, aumentaron las movilizaciones estudiantiles en diferentes ciudades y se ocuparon varias universidades. Si en febrero de 1968 había 19 universidades ocupadas, se estima que tres meses más tarde, en mayo, a excepción de la Università Bocconi de Milán, todas las universidades italianas habían sido ocupadas en algún momento.

El creciente malestar y la movilización estudiantil respondían a una serie de factores estructurales. En la década de los sesenta, la universidad italiana había cambiado. Se había asistido a un constante proceso de democratización de la enseñanza. Se hablaba de un auténtico boom de la demanda de formación superior. Ya no representaba un lugar elitista, reservado a las clases adineradas del país. La universidad italiana había pasado a ser una «università di massa», con un gran aumento del número de alumnos matriculados.

Este rápido crecimiento de los inscritos exigía una serie de cambios en varios ámbitos: profesorado, tipo de formación e infraestructuras entre otros. No obstante, el proceso de adecuación de la nueva universidad a las nuevas exigencias fue lento e insuficiente. Al aumento del número de alumnos no 
correspondió un incremento del profesorado ni la mejora de las infraestructuras. Dato emblemático es el caso de la Università di Roma donde se estima que en 1968 su estructura estaba preparada para albergar unos diez mil estudiantes, frente a los casi sesenta y tres mil matriculados. Pero no era solo un problema de aulas sobrepasadas; a esto se le sumaban las críticas por el tipo de educación que se impartía. Se consideraba que no proporcionaban una enseñanza acorde con los tiempos ni preparaban a los jóvenes para su futura inserción en el mercado laboral. El contenido de la docencia se consideraba anacrónico y alejado de la realidad. La insatisfacción ante la formación universitaria se hacía evidente en lo concerniente a los programas de estudios. Mientras por las calles los contestatarios mitificaban figuras como Che Guevara, Mao u Ho Chi Minh, los programas de las asignaturas de política o de pensamiento político alcanzaban cronológicamente a autores como Montesquieu o Rousseau. En los programas más actualizados se estudiaba la revolución francesa. Era insuficiente, los jóvenes deseaban leer a Marx y a Lenin, a Sartre y a Marcuse.

El malestar estudiantil y las expectativas frustradas se enmarcaban en un proceso de reflexión sobre el rol de la universidad en la sociedad. En esta óptica, se criticaba la propuesta de reforma universitaria del ministro democristiano Luigi Gui por favorecer mecanismos de selección social y primar una visión economicista del saber al subordinar la educación a los intereses de la economía de mercado. De esta forma, la crítica estudiantil era una crítica a la totalidad del sistema universitario, que ponía en tela de juicio su papel en la sociedad a la vez que se reclamaba mayor poder y protagonismo para los estudiantes. Por estos objetivos se desplegó desde el otoño de 1967 una movilización estudiantil que, «partiendo de individuales contextos de conflicto» en pocos meses se extendió por todo el territorio nacional «contra la instrucción universitaria, contra las autoridades académicas, contra la forma de trasmisión del saber y la calidad de los mismos saberes transmitidos» (Tolomelli, 2015: 103).

Los estudiantes no cuestionaban solo el sistema universitario. Era algo más que una revuelta generacional. Era una protesta más profunda que envolvía una severa crítica del sistema socio-político y cultural. En Italia se había asistido a una modernización sin reformas y en el marco de un sistema político bloqueado (Höbel, 2004: 423), caracterizado por la invencibilidad de la Democracia Cristiana y por el carácter subalterno del Partido Comunista italiano. Así, las ocupaciones permitieron «al movimiento enriquecer y reforzar su heterogénea proyección ideal y sus prácticas organizativas», yendo más allá de una dimensión sindical para «convertirse en una contestación del sistema no solo universitario, sino también político, social y cultural» (Di Maggio, 2014: 97). 
Eso explicaría también el apoyo que recibió el movimiento estudiantil por parte de los obreros y proletarios italianos. Compartían un profundo malestar social ante la nueva Italia de los sesenta; un país donde el llamado milagro económico no estuvo exento de críticas. La mejora de las condiciones económicas mostraba sus límites. No era generalizada ni suponía la resolución de los viejos problemas socio-políticos. Incluso provocaba una serie de consecuencias que debían ser objeto de mayor atención por parte de los políticos, tales como la inmigración masiva del sur al norte industrial (Ginsborg, 2003: 219) o las condiciones de los obreros, sobre cuyas espaldas recayó el precio de la desaceleración después de los años de crecimiento sostenido.

En este escenario de crisis, los acontecimientos de Valle Giulia en Roma representan el momento de mayor interés mediático y político por las movilizaciones estudiantiles. El 1 de marzo de 1968, tras la desocupación de la Facultad de Arquitectura de la Universidad La Sapienza en Roma, se asistió a una nueva manifestación de estudiantes. La situación degeneró en la explanada de Valle Giulia en la que se encontraba la facultad y donde se produjeron unos enfrentamientos muy violentos entre la policía y los estudiantes. El balance del choque resultó especialmente duro. Aunque no hay acuerdo sobre las cifras, se habló de cientos de heridos (casi 150 entre la policía, más de 450 entre los estudiantes). Las imágenes de gases lacrimógenos y de los coches de policía incendiados recorrieron el mundo. Hubo una decena de detenidos. Las escenas de la batalla campal de Valle Giulia se convirtieron en un símbolo de la rebelión juvenil del 1968. Se habló de la «batalla de Valle Giulia»³ . Era la primera vez que los manifestantes respondían de una manera tan impetuosa. Y era la primera vez que los medios de comunicación recurrían a expresiones belicistas: enfrentamiento, guerra, choque, violencia.

El acontecimiento provocó reacciones opuestas: una parte de los medios apuntaron a la radicalización del movimiento y su uso de la fuerza como parte de una estrategia revolucionaria; otros condenaron la actuación policial, mostrando su solidaridad con los jóvenes. En este escenario, Pier Paolo Pasolini, cineasta e intelectual italiano cercano al PCI, escribió una larga poesía titulada «Il Pci ai giovani» ${ }^{4}$. En el texto, Pasolini expresaba su sorpresa por los hechos y también su disconformidad: «En Valle Giulia, ayer, se produjo un episodio de lucha de clases: y vosotros, queridos (si bien estabais de la parte de la razón)

3. BULTRINI, Giampaolo; SCIALOJA, Mario. «La battaglia di Valle Giulia». L'Espresso. 10-31968. <http://temi.repubblica.it/espresso-il68/1968/03/10/la-battaglia-di-valle-giulia/>, [15.12.2019].

4. PASOLINI, Pierpaolo. «Il Pci ai giovani». Espresso, 16-6-1968. <https://temi.repubblica. it/espresso-il68/1968/06/16/il-pci-ai-giovani/>, [15.12.2019]. 
erais los ricos. Mientras los policías (que iban con la parte equivocada) eran los pobres. ¡Bella victoria, por lo tanto, la vuestra! En estos casos, a los policías se les dan flores».

En su opinión, durante estos enfrentamientos entre policía y estudiantes, se estaba asistiendo efectivamente a un episodio de lucha de clases, cuyos papeles estaban invertidos, ya que los estudiantes eran la clase alta mientras la policía la baja. Los estudiantes sin conciencia de clase pretendían el cambio sin comprender quien era realmente su enemigo. «Aunque de la parte de la razón», su «sagrado vandalismo» iba en la dirección equivocada: «Los policías son hijos de los pobres. Vienen de periferias». La postura de Pasolini ha sido objeto de varias interpretaciones. La más difundida considera que el autor simpatizó abiertamente con los policías. Se omite la comprensión de la segunda parte de la poesía, en la que el intelectual escribía: «Mirad como los visten: como payasos, con esa tela áspera que huele a rancio, furriel y pueblo. Lo peor de todo, naturalmente, es el estado psicológico al que los han reducido (por cuarenta mil liras al mes): sin más sonrisa, sin más amistad con el mundo, separados, excluidos (en una exclusión sin igual); humillados con la pérdida de la condición humana por la de policías (ser odiados provoca odio). Tienen veinte años, vuestra edad, queridos y queridas». Palabras duras, de compasión que, como el resto de la poesía, pretendían concienciar a los estudiantes, invitándoles sutilmente a abandonar su postura pequeño-burguesa para ir hacia el PCI, converger con los obreros para una lucha de clase más autentica.

Con anterioridad a dicho suceso, el interés por las movilizaciones era limitado y fue aumentando con el paso del tiempo. En términos generales, en sus inicios la contestación estudiantil generó escaso interés mediático y político. A excepción del PCI los partidos políticos se mostraron favorables al uso de la fuerza para reprimir dichas manifestaciones, mientras tendían a infravalorar este suceso, tachando a los estudiantes de jóvenes rebeldes, propensos a la agitación y a la desobediencia.

La postura mayoritaria de la prensa italiana fue crítica con el movimiento. Se hablaba de «agitación», «revuelta», «ocupaciones». Casi siempre se refería al movimiento estudiantil en términos denigratorios. Se subrayaban las divisiones en el movimiento, la falta de objetivos comunes, la desorganización de sus actividades y las acciones violentas. «Los estudiantes -en cuanto jóvenes-eran considerados utopistas» (Colanicchia y Russo Spena, 2018: 149). A veces se mostraba un talante condescendiente en el marco de una postura paternalista. Una pluma hiriente y mordaz como la de Indro Montanelli describía así el movimiento: "Sin embargo debajo de su 'revolución cultural', pese al imponente y complicado vocabulario sociológico del cual beben, mi nariz advierte 
hedor más de Bakunin que de Mao, y de un Bakunin interpretado a la italiana, es decir como coartada ideológica para [...] la confusión, el caos, un 'cuarenta y ocho'» ${ }^{5}$.

Las protestas estudiantiles tuvieron particular relevancia en L'Unità, el periódico fundado por Antonio Gramsci en 1924 y desde su nacimiento estrictamente ligado al PCI. Tras un primer desinterés por el tema, el diario cambió de actitud y asumió un doble cometido: informar sobre los hechos denunciando la represión gubernamental, a la vez que expresaba su solidaridad con la lucha estudiantil. En enero, publicó en primera página un artículo sobre la intervención de Alessandro Natta en la Cámara criticando la Ley Gui. El político del PCI consideraba que «la reforma no corresponde a las exigencias $\mathrm{y}$ a las aspiraciones del mundo universitario y de la sociedad italiana» $\mathrm{y}$, sobre todo, añadía que «no son los comunistas los que alimentan la protesta de los estudiantes; ellos la apoyan, pero en realidad son los estudiantes mismos y parte de los docentes los que entienden que deben manifestar la necesidad de una renovación radical». Terminaba afirmando que «a las ansias de los estudiantes» se respondía «con una toma de conciencia de la clase dirigente» ${ }^{6}$. El diario se mostraba comprensivo con las actuaciones estudiantiles y reflexionaba sobre el eco de sus protestas: «Los ateneos son el terreno de los enfrentamientos civiles a altísima tensión y a altísima escala. No una ley sino el enfrentamiento cultural y social está liquidando a la vieja universidad $»^{7}$.

\section{El PCI y el movimiento estudiantil}

La relación con el movimiento estudiantil constituye uno de los momentos más intensos en la dialéctica política interna del Partido Comunista italiano durante la secretaría de Longo, pues remite a la cuestión general de «las difíciles relaciones -y al tema de la ruptura/continuidad- entre 'izquierda histórica' y 'nueva izquierda'» (Höbel, 2004: 419).

Como en el caso de L'Unità, la postura del PCI fue evolucionando. Desde finales de 1967, las reivindicaciones de los estudiantes y sus luchas entraron en su agenda política. El partido cuestionaba sobre todo la represión y las actuaciones policiales, expresando su solidaridad a los estudiantes y docentes. Sin embargo, en el partido se emprendía un intenso debate sobre cómo mejorar sus condiciones, se cuestionaba la Ley Gui y las actitudes del gobierno en un claro

5. MONTANELLI, Indro. «Gli altri giovani». Corriere della Sera, 6-3-1968.

6. «Alle ansie del mondo universitario rispondere con una vera riforma». L'Unità, 12-1-1968.

7. ROSSANDA, Rossana. «Nel vivo di una discussione che scuote gli atenei». L'Unità, 10-2-1968. 
intento de asimilar el recién configurado movimiento estudiantil a su política de oposición al gobierno. Se trataba de una difícil «integración»: el movimiento estudiantil mostraba un fuerte componente reivindicativo y una postura crítica con todo el sistema. No se libraba el PCI. Por eso, los primeros intentos de aproximación del partido encontraron la resistencia del movimiento estudiantil que se mostraba insatisfecho con el PCI, apelando a una autogestión al margen de cualquier fuerza política. El movimiento deseaba mantener sus márgenes de autonomía y su postura era crítica hacia la política y sus intérpretes.

La postura del PCI oscilaba entre el reconocimiento de la fuerza propulsiva del movimiento y el temor a la asunción de posturas extremistas. Entre la apertura y la polémica. Entre el diálogo y la demonización. Compartía la crítica hacia la propuesta de la nueva ley universitaria, proclamando «meglio nessuna legge che una cattiva legge». Pese a reconocer el carácter positivo de la protesta, al Partido le costaba comprender la situación. En palabras de Rossana Rossanda «las hegemonías no se imponen, se conquistan»: «no se trata de instrumentalizar una lucha, ni de reducir[la...] sino de entenderla y de entender los nudos y de señalar el camino $»^{8}$.

En la primavera de 1968, era evidente dentro del partido la presencia de dos posturas antagónicas: por un lado quienes como Giuseppe Chiarante, consideraban el malestar juvenil expresión de un descontento político, demanda de «una sociedad diferente» al margen de la esperanza reformista (1968: 56-59); y, por otro quienes les consideraban una «molestia», como Gian Carlo Pajetta ${ }^{9}$, y les acusaba de abogar por formas de protesta anarquistas, mirándoles con recelo en cuanto partidarios de una renovación inmediata y radical.

$\mathrm{Al}$ intensificarse la protesta juvenil, el PCI reflexionó más detenidamente sobre su valor y las posibles implicaciones políticas. En el Comité Central del 16 y 17 de enero de 1968, era evidente la preocupación por la posible aparición de formaciones a la izquierda del Partido. Se rumoreaba acerca de la creación de una formación política o incluso de un partido revolucionario posicionado políticamente a la izquierda del PCI. Esta formación/partido podría encontrar el apoyo de los estudiantes, recelosos hacia el partido y deseosos de un proyecto nuevo más acorde con sus demandas y también de los militantes del partido que consideraban que el PCI descuidaba temas como la solidaridad con Vietnam o la revolución cultural china.

Desde febrero de 1968, dentro de la Dirección de PCI era evidente la dificultad para relacionarse con este nuevo escenario, para comprender plenamente

8. ROSSANDA, Rossana. «Un successo per l'università». L'Unità, 27-10-1968.

9. PAJETTA Gian Carlo. «I giovani non sono una 'difficoltà', ma sono un problema». Rinascita, 10-03-1967. 
su amplitud y variedad. Reconocía la necesidad de una renovación intelectual, moral, cultural y política del país (Napolitano, APCI, 1968: 555-556), aunque la cuestión central en el partido era qué vinculación establecer con el movimiento de protesta universitario. Así mientras el PCI era partidario de convencer a los estudiantes para que confluyesen en la lucha general para transformar la sociedad, dictando las condiciones de dicha confluencia, los estudiantes cuestionaban los «retrasos del partido» y le invitaban a modificar su forma de relacionarse con un nuevo sujeto/movimiento.

Se trataba de posiciones críticas pero abiertas, que se acercaron después de los acontecimientos de Valle Giulia: pese a la presencia de voces críticas, el PCI condenaba la intervención policial y en un comunicado de la Dirección del partido reclamaba «la afirmación de la figura protagonista del estudiante» (AA.VV., 1969a: 409-410). El mismo día de los hechos, Paolo Bufalini publicó un artículo en Rinascita, en el que reconocía el carácter progresista del movimiento ${ }^{10}$.

Aunque las críticas se mantuvieron ante la posible radicalización del movimiento, se asistió a un acercamiento del partido ante al aumento de la represión y de los ataques neofascistas. Si para Claudio Petruccioli, el partido debía «actuar dentro de este movimiento siguiendo su lógica» ${ }^{11}$, para Giorgio Amendola el principal peligro era «quedarse mirando o adecuarse a su espontaneidad», invitando a una "presencia activa de nuestro partido dentro del movimiento $»^{12}$.

En términos generales, el PCI era partidario de una «confluencia» del movimiento estudiantil en el partido. La hegemonía de la lucha seguía cayendo sobre la clase obrera, pero se auguraba un respaldo de los estudiantes, una convergencia de sus demandas en el marco de una lucha general. Por su parte, los estudiantes deseaban «salir de las aulas universitarias» y convertir su protesta en una contestación global al sistema. En este debate prevaleció la línea marcada por el Secretario General del PCI, Luigi Longo, favorable a establecer una relación constructiva entre partido y estudiantes. Su actitud se hizo manifiesta ante el Comité Central del 27 de marzo de 1968, en sus encuentros con exponentes de la movilización estudiantil en abril o en su artículo de mayo titulado «Il movimento studentesco nella lotta anticapitalista».

En su informe ante el CC, Longo afirmaba que «ninguna transformación, por modesta que sea, puede tener éxito sino como momento y aspecto de una acción más amplia que afecte y ponga en discusión el sistema mismo en su

10. BUFALINI, Paolo. «Il partito e gli studenti». Rinascita,1-3-1968.

11. «Si riunisce il Comitato centrale del Pci; all'ordine del giorno la posizione di fronte al movimento degli studenti». L'Unità, 28.03.1968.

12. L'Unità, 29.03.1968. 
conjunto» ${ }^{13}$. Y en el artículo de mayo ${ }^{14}$, argumentaba que la lucha estudiantil pertenecía a un movimiento reivindicativo más amplio y se sumaba a otros, ya que su lucha se correspondía «con toda la temática y con todos los problemas del movimiento obrero italiano». El artículo se dirigía tanto al partido como al movimiento. Longo consideraba al movimiento como «un interlocutor no solo válido, sino necesario», portador de una experiencia valiosa. El Secretario argumentaba que la situación y la problemática estudiantil podía ser compartida con el movimiento obrero italiano y no debía considerarse un movimiento sectorial. Era legítimo que los estudiantes se preocupasen de sus condiciones materiales, pero también que anhelasen a comprender «los problemas de la revolución italiana». Su objetivo con esa actitud «abierta», dialéctica entre partido y movimiento estudiantil, era tanto neutralizar la preocupación existente en el partido, como evitar la posible masiva abstención de los estudiantes, invitándoles a pasar del «voto en blanco» al «voto rojo». Longo aspiraba a recuperar el espacio político a la izquierda del partido y certificaba un cambio de actitud: si con anterioridad las relaciones con los estudiantes venían filtradas por la Federazione Giovanile Comunista Italiana (FGCI), en este nuevo escenario el partido se convertía en interlocutor directo, proclamando la convergencia en la lucha con los estudiantes. La línea de Longo de que no se trataba de un movimiento sectorial, sino de un actor en la lucha al que convenía secundar en algunos aspectos para que sus reivindicaciones pudieran combinarse con la del movimiento obrero fue la que prevaleció.

Ante las elecciones de mayo de 1968, el movimiento estudiantil «estaba dividido entre la 'scheda rossa' (es decir voto a PCI o PSIUP ${ }^{15}$ ) y el voto anulado con una escrita revolucionaria» (Flores d'Arcais, 2018: 132). Por eso, el PCI intentó priorizar la búsqueda de una sintonía entre el partido y los movimientos de protesta, presentándose como una creíble alternativa anti sistema. En vísperas de las elecciones no parecía conveniente tachar la revuelta de extremismo, pese a las críticas abiertas a la radicalización de sus gestos.

Según varias lecturas, el artículo de Longo determinó una tregua en la difidencia mutua entre el partido y el movimiento estudiantil, apostando por una posición dialéctica. Dicha actitud, definida como la «strategia dell'attenzione» (Di Maggio, 2014: 101), contribuyó al éxito electoral del 19-20 de mayo, cuando el PCI incrementó sus votos tanto en la Cámara (de 25,3\% a 26,9

13. LONGO, Luigi. «È ora di cambiare. Rapporto alla sessione di CC e CCC del PCI del 27-28 marzo 1968». L'Unità, 27-3-1968. 8-9.

14. LONGO, Luigi. «Il movimento studentesco nella lotta anticapitalista». Il Contemporaneo (suplemento de Rinascita), 3-5-1968.

15. Partido Socialista Italiano de Unidad Proletaria. 
por ciento) como en el Senado (junto con el PSIUP obtuvo el 30\%). En esta circunstancia era evidente que el PCI había conseguido captar el voto juvenil.

\section{Influencia y repercusiones del mayo francés en Italia}

El movimiento estudiantil francés estalló más tarde, pero con una intensidad mayor. Mayo de 1968 fue un momento/movimiento insurreccional, capaz de sacudir profundamente a la República francesa. Las ocupaciones y manifestaciones estudiantiles, la huelga general, el difundido malestar nacional más que polarización del país, provocaron su paralización. De una reivindicación se pasó a una enmienda a la totalidad del sistema. Pese a su fugacidad, los casi sesenta días que hicieron «temblar» al país galo entraron de lleno en el imaginario colectivo italiano. Los episodios franceses contribuyeron a la difusión de la percepción que había estallado el «mayo rojo» (Flores d'Arcais, 2018: 132). Las crónicas de las ocupaciones, de las barricadas del barrio latino, de la huelga general, conmocionaron a una generación, convirtiéndose en mito desde su aparición.

Los jóvenes italianos y franceses compartían un sentimiento común: el ansia del cambio. Sin duda, el mayo francés interesó e influyó en los jóvenes italianos más que cualquiera de los otros acontecimientos del mismo momento (la Primavera de Praga, la matanza de México, la movilización estudiantil en Estados Unidos). Y eso no dependió solo de la mayor proximidad geográfica, las similitudes socio-económicas, la proximidad cultural o la presencia de los partidos comunistas más fuertes de Europa occidental. La razón principal residió en el mencionado deseo de modificar el sistema de manera profunda y radical. Los sucesos franceses fueron capaces de avivar la pasión revolucionara. En el imaginario de los jóvenes italianos, las barricadas francesas abrían el camino a la posibilidad del triunfo de la revolución. La revolución, verdadero mito y punto de llegada para los estudiantes italianos, determinaría el consecuente cambio de la sociedad. Por la vía violenta, derrumbando un sistema considerado podrido, su triunfo era la plena realización de la utopía estudiantil.

La contestación estudiantil en Francia provocó una profunda crisis sociopolítica. El sistema se tambaleó y la huelga general, en la que participaron nueve millones de trabajadores, una de las mayores de la historia, fue una señal evidente de que el malestar traspasaba las aulas universitarias. Ante este panorama, en Italia se debatió sobre las posibilidades de éxito de la revolución; sobre si un suceso con estas características fortalecía o debilitaba la posibilidad de un cambio revolucionario en un país de capitalismo maduro como Francia, no tan diferente a Italia. Es decir, los jóvenes y los intelectuales de izquierda se preguntaban si se trataba de un modelo a imitar o a evitar. En los círculos 
culturales e intelectuales italianos cercanos a la extrema izquierda, se asistió a una idealización de Francia como «depositaria de las esperanzas revolucionarias» y se mitificó el suceso, más allá de sus verdaderos logros. Se le describía como la sublimación de la experiencia de la Comuna de Paris de 1871.

A diferencia del caso italiano, el mayo francés contó con una «mitizzazione in corso d'opera». Por su capacidad de «transposición de un imaginario filtrado por un estilo innovador de comunicación política e información militante» (Benci, 2009), se demostró un formidable productor de cultura. Destacó su enorme producción iconográfica: sus fantasiosos eslóganes, sus llamativos grafitis, sus expresiones ácratas que conquistaron el imaginario colectivo mundial. Italia no fue inmune de esta fascinación: se importaron sus eslóganes, se copiaban sus símbolos. Ejemplo de esta gran fascinación del mayo francés fue la creación de varias organizaciones y formaciones cuyos nombres u objetivos coincidían con homólogas galas como Lotta Continua («nacionalización» de lutte continue), Avanguardia Operaia, Potere Operaio que tuvieron una relación difícil con la «izquierda oficial».

Desde el punto de vista dialéctico, el 1968 italiano creó sus propios eslóganes como «siamo tutti proletari» (somos todos proletarios), «prendiamoci la città» (tomemos la ciudad) o «fantasia al potere» (fantasía al poder), deudores del estilo breve, conciso y alegórico del mayo francés. El movimiento estudiantil italiano también se caracterizó por su lucha antifascista y el enfrentamiento con formaciones de extrema derecha. Por esa razón también recurrió a eslóganes violentos como: «fascisti, borghesi, ancora pochi mesi» (fascistas, burgueses, aún pocos meses) o "uccidere un fascista non è reato» (matar a un fascista no es delito). Y, desde el punto de vista gráfico, contaba con una variedad de carteles inspirados en la iconografía parisina, mostrando nuevamente la «deuda de reconocimiento» al mayo francés. Carteles que evocaban el ideal de estar viviendo el «sueño de la revolución de mayo». Una fantástica utopía. Se aludía a la revolución en marcha y al mito del retorno a la Comuna de Paris. Se apelaba a una continuidad entre el sueño y la realidad, entre el imaginado y la imagen real, enfatizando su mezcla de respeto y deuda a Francia, pendientes de su porvenir y sus consecuencias. Un suceso retransmitido en directo, vía radio, pero que aun así se prestaba a interpretaciones, adaptaciones o incluso distorsiones sobre la «marcha».

\section{El 1968 desde una perspectiva crítica}

Al estallar el movimiento estudiantil de 1968, sorprendió su fuerza desestabilizadora, señal de que no se trataba de un fenómeno repentino. Y resulta limitativo considerarlo solo como expresión de un malestar juvenil: el movimiento 
de 1968 en Italia demostró una particular interacción con otro actor social, el movimiento obrero. En términos gramscianos, se hablaba de una alianza entre estudiantes y obreros para obtener una nueva Hegemonía.

Una de las preguntas típicas que suele plantearse es por qué las protestas estudiantiles despertaron tanto interés en el PCI. Es probable que inicialmente, el objetivo fuera comprender el contenido de la protesta más allá del rechazo total a la nueva ley universitaria. Pero pronto comprendieron que su malestar iba más allá. El partido miró a los jóvenes con gran interés no solo por posibles cálculos electorales. Había otra razón: en un momento de estancamiento y de bloqueo político, el partido vio como desde fuera podía llegar una "chispa», un sujeto socio-político nuevo que le invitaba a reflexionar sobre su papel, su estrategia y sus objetivos. Dentro del PCI, había quien consideraba a los estudiantes como un valor añadido y un estímulo nuevo para tantear nuevos caminos, nuevas reivindicaciones.

La fuerza y propagación del movimiento fue inesperada para el PCI. Eso explica la dificultad y el retraso con el que el partido se planteó como relacionarse con ellos. Y al menos en los primeros meses, el PCI mostraba la dificultad de captar las instancias de la protesta. No comprendía que el movimiento era el reflejo de la tensión que se vivía en la sociedad italiana. Su demanda de renovación de la sociedad debía suponer una reflexión del partido acerca de su relación con la sociedad. La preocupación por las elecciones de mayo de 1968 condicionó la comprensión del alcance de la protesta.

Por primera vez desde la posguerra, el PCI sintió el temor de perder su hegemonía en la izquierda política italiana, advirtiendo «la exigencia de una lucha 'en el frente de izquierda'» (De Luca, 1968: 38). A su izquierda, apareció una izquierda beligerante y especialmente crítica con su actuación política. En estos círculos, se argumentaba que la actitud del PCI era la demostración de que sus intenciones y capacidad revolucionarias se habían diluido. Hablaban de «estancamiento del espíritu revolucionario». Tanto era así que en los documentos oficiales el partido omitía la palabra revolución, prefiriendo «expresiones más edulcoradas como 'renovación democrática' o al máximo 'transformación socialista'» (Coccia, 2008: 148).

Los sucesos franceses representaron una oportunidad para reflexionar sobre la naturaleza revolucionaria de los movimientos sociales. A la vez, invitaban a considerar desde una nueva perspectiva los tiempos de transición al socialismo. Y, sobre todo, el mayo francés con sus peculiaridades y consecuencias, representó una ocasión de confrontación entre la izquierda oficial (el PCI) y las vanguardias juveniles. Estas últimas se mostraban divididas entre quienes eran partidarios de converger en el partido creando una unión de las izquierdas 
y aquellos que cuestionaban la postura del PCI (asimilándola a la del PCF), criticando el abandono de la vía revolucionaria.

A pesar de mostrar una atención hacia las reivindicaciones del movimiento estudiantil mayor que cualquier otro partido, parte de los jóvenes consideraron que la postura del PCI se asemejaba a la tan criticada del PCF. Tenían la impresión de que el partido quería beneficiarse del movimiento como «arma arrojadiza» contra la DC. El PCI aceptaba con dificultad que se trataba de un movimiento social con unas características propias que prefería organizarse bajo el lema de la autogestión antes que encuadrarse en el partido. Y, sobre todo, que tenía un objetivo: ejercer un papel destacado fuera de las aulas universitarias. En este escenario crítico, Pietro Ingrao y Rossanda fueron dos excepciones, mostrándose los más hábiles para entrar en sintonía con el movimiento. Y eso se debió al hecho de que ambos políticos se esforzaron por comprender sus demandas. No cayeron en la trampa de encasillarles, ni de minimizar sus reivindicaciones.

Los jóvenes que se mostraron más críticos con el PCI recurrieron a una acusación clásica: reprochar al partido haber sustituido el valor de la revolución por el de la elección, de haberse convertido en un engranaje más dentro del régimen. De ahí la fascinación italiana por el mayo francés, por su apuesta por la vía revolucionaria como estrategia de derrumbe del sistema y apertura del camino a un nuevo escenario. La renovación propuesta por el Partido no era tan inmediata ni tan radical como ellos querían. Siempre en esta línea, los estudiantes -al menos aquellos más próximos al PCI- criticaban la disponibilidad e interés del partido en debatir temas actuales para el movimiento como la revolución cultural china, la experiencia cubana, la situación de América Latina y más en general, los problemas relacionados con la «estrategia revolucionaria» y la vía italiana al socialismo.

No cabe duda que «el 68, annus mirabilis de la revuelta de los estudiantes, no fue una breve llama aislada en ningún lugar: con intensidad, duración y amplitud diferentes [...] constituyen un verdadero 'periodo de los movimientos'» (Grispigni, 2018: 101). En lo que concierne a la relación entre los dos movimientos estudiantiles, podemos establecer una serie de elementos comunes: ambos intentaron construir un frente común con el movimiento obrero y desconfiaron del partido comunista (en Francia más que en Italia); adoptaron acciones comunes y fueron protagonistas de episodios violentos con las fuerzas del orden; abogaron por formas de autogestión y por la ocupación de las universidades. En cada Universidad, creaban su «propio 'parlamentino estudiantil', dentro del cual se desplegaban conflictos y bandos de manera análoga a cuanto ocurría en ámbito político» (Tolomelli, 2015: 108). Entre los dos movimientos 
-y en términos generales con los demás-, «hay un punto de conexión en la toma de conciencia de la problemática del poder, en el proyecto utópico de forzar los tiempos de la historia» (Santarelli, 1997: 151). Sus críticas del sistema universitario conllevaban la difusión de un discurso igualitario, antiimperialista y anticapitalista. Se cuestionaba la sociedad industrial capitalista -y el papel que otorgaba a la Universidad-a la vez que empezaba la crítica a la alternativa soviética: la URSS ya no representaba un modelo atractivo. Y mientras perdía su poder de atracción, otras experiencias en el entorno comunista seducían a los jóvenes franceses e italianos: la revolución cultural china, la guerrilla vietcong, la guerra de liberación argelina. Fidel Castro y Che Guevara fascinaban a las nuevas generaciones con sus experiencias revolucionarias.

Los jóvenes italianos y franceses mostraban su malestar contra el sistema. Como muestra, en el caso italiano pese a que la reforma Gui no se aprobó y tuvo un iter parlamentario especialmente accidentado, las protestas no solo no disminuyeron, sino que se extendieron por todo el país. En Italia y en Francia, reprochaban una gestión autoritaria del poder por parte de las instituciones nacionales. Los estudiantes se oponían a las estructuras de poder existentes, promoviendo una visión antiautoritaria de las relaciones, que iba más allá de la rebeldía. Era también una crisis de representación. Leían El hombre unidimensional de Marcuse y sobre todo el célebre libro de Lenin ¿Qué hacer?, que, aunque escrito entre1901 y 1902, les parecía poder ser útil en su contexto. Y, si en Francia se popularizó la lectura del libro rojo de Mao, asequible a un franco veinte, en Italia triunfaba la lectura de Gramsci y su teoría sobre la Hegemonía. Los jóvenes italianos hicieron suya la cita: «un grupo social puede y aún más debe ser dirigente ya antes de conquistar el poder gubernamental (es esta una de las condiciones principales para la misma conquista del poder); después cuando ejerce el poder, aunque lo tenga fuertemente en un puño se convierte en dominante, pero debe continuar siendo también dirigente» (1975: 2010-11).

\section{El PCI ante el 1968 italiano y el francés}

La relación del Partido Comunista italiano con el movimiento estudiantil italiano fue «de odio y de amor» (Chiarante, 1996: 129). Al igual que el PCF, la relación del partido con los nuevos movimientos sociales resulta algo contradictorio. Especialmente con el movimiento estudiantil. Como se ha indicado con anterioridad, le preocupaba su carga anti-sistema y su crítica de la identidad comunista, a la vez que le fascinaba su vitalidad y poder de atracción de la parte apática de la sociedad. Por esas razones, los consideraban como un activo que debía ser controlado en el marco de la estrategia del partido. Y por esa misma desconfianza-atracción, en la retórica del Partido y en la propaganda electoral 
el PCI seguía privilegiando las organizaciones tradicionales del movimiento obrero, dando poco espacio y peso al movimiento estudiantil. En los carteles electorales de 1968 apenas había referencia a este movimiento.

A medida que la movilización estudiantil se fue intensificando y pasó a ser una «contestación global», creció el interés del partido. El PCI percibía la necesidad de modificar su relación con los movimientos sociales y su presencia en aquellas formas de protesta y movilización que estaban fuera de su control. En el partido se hacían básicamente dos preguntas: la primera acerca de la verdadera capacidad de los jóvenes de desenvolver un papel determinante también fuera de las aulas universitarias. En cuanto sujeto socio-político «nuevo», se reflexionaba acerca de su peso electoral y sobre su capacidad de emprender una crisis potencialmente revolucionaria. Y la segunda, era sobre la misma organización de los estudiantes y su posible integración en el partido. Pese a la presencia de voces y medios -diarios como Ottobre- que auguraban un pacto entre obreros y estudiantes, el PCI quería comprender el margen de actuación del movimiento y el valor que daba a la autogestión, fuera del partido.

El PCI se interrogaba acerca de los posibles efectos del mayo francés sobre el movimiento estudiantil italiano. Temía que pudiera convertirse en un modelo a seguir y que tanto la radicalidad de sus planteamientos como las incomprensiones entre el movimiento y el partido pudieran traspasar fronteras. En su debate interno, eran varios los temas objeto de controversia. Entre otros: el valor de los nuevos movimientos sociales, si representaban una potencial amenaza o podían constituir un recurso; qué estrategia se debía promover ante estos movimientos; y, por último, el valor de la lucha, cuestionando la centralidad del elemento reivindicativo a favor de una perspectiva gradualista.

El mayo francés aun siendo un acontecimiento externo fue asumiendo importancia interna, calándose en el debate entre dos visiones enfrentadas: por un lado, aquellos que le consideraban como un «mítico 1905», es decir un suceso previo, antesala de una inminente «revolución de octubre»; y por el otro, aquellos que consideraban estos sucesos como una prueba de fuego para la que el PCI debía preparase. En este debate, el secretario Longo decidió asumir una posición autocrítica, manifestando la existencia de una brecha entre el partido y la situación estudiantil. Es más, lamentaba una distancia respecto a las preocupaciones de los jóvenes. Invitaba a una lucha conjunta, destacando la capacidad del PCI de movilizar a las masas.

La reunión de Dirección del 6 de junio se centró en realizar un análisis de los hechos franceses. El tema sirvió como ocasión para reflexionar sobre la naturaleza revolucionaria de los movimientos sociales de la época y sobre las formas y los tiempos de transición al socialismo. En el partido existían dos 
posturas respecto a la relación PCF-estudiantes. De una parte, políticos como Petruccioli, Ingrao o Rossanda especialmente críticos con el PCF, considerando perjudicial el enfrentamiento frontal con el movimiento estudiantil. Petruccioli estimaba que en el caso de Francia se había llegado al «umbral de una situación revolucionaria» sin haber previamente comprendido como enfrentarse a dicho umbral. Ingrao argumentaba que la radicalidad del movimiento francés ponía de manifiesto las profundas contradicciones de los países capitalistas, lo que hacía posible la apuesta inmediata, en estos países, por una estrategia anticapitalista. Ingrao criticaba al PCF su escasa capacidad de conectar con el nuevo movimiento, su ineptitud en reconducir las relaciones hacia una nueva unidad.

Frente a la izquierda del partido, se posicionó el ala conservadora del mismo, formada por Amendola, Napolitano y Bufalini. Acusaban a parte del partido de sufrir la fascinación por estas «explosiones de extremismo». Los exponentes de esta postura se mostraban especialmente críticos en cuanto temían tanto una posible división interna del partido como un cambio en los equilibrios internos dentro del PCI. Por eso, intentaban «rectificar» la línea marcada por Longo que se caracterizaba por el diálogo y el respeto hacia el movimiento estudiantil.

Dentro del ala de «derecha» del PCI, tuvo particular relevancia un artículo de Giorgio Amendola ${ }^{16}$, que realizaba una atenta autocrítica de la estrategia del PCI en la relación con el movimiento estudiantil. El centro de esta autocrítica no residía en el déficit organizativo del partido, sino en su incapacidad de conquistar la hegemonía en los movimientos de lucha. Afirmaba que el partido mostraba su «debilidad al conducir una lucha contra las posiciones extremistas y anarquistas afloradas en el movimiento». El dirigente napolitano consideraba necesario luchar contra las formas de sectarismo, oportunismo y extremismo, aproximándose al planteamiento de la mayoría del PCF. Estimaba peligrosa la postura de aquellos que pretendían construir la hegemonía a través de una actitud condescendiente con los extremistas. Era oportuno recordar tanto la «función hegemónica de la clase obrera en las luchas para el socialismo» como el papel del partido. Para Amendola, la fascinación de las tesis de Marcuse y de Mao en su versión vulgarizada-estudiantil no difería de las antiguas formulas anarco-sindicales, antinacionales, que, en lugar de unir al movimiento obrero, le condenan a la derrota. Por esta razón, defendía la postura del PCF sin reservas, subrayando la dificultad de enfrentarse simultáneamente al anticomunismo de los reaccionarios y de los grupos extremistas que ponían al mismo nivel el imperialismo y el socialismo francés.

16. AMENDOLA, Giorgio. «Necessità della lotta su due fronti». Rinascita, 7-6-1968. 
Es preciso recordar que la división interna dentro del PCI se remonta a los últimos años de la secretaría de Togliatti, cuando afloraron diferentes posturas dando lugar a una animada dialéctica interna. Aunque las diferencias se agudizaron en los años siguientes, el bienio 1962-1964 representa un 'antecedente' esencial (Höbel, 2010: 23). Longo, partidario de una diferente concepción de la secretaría, declaraba: «Yo debo ser el secretario general recogiendo de todos lo que luego debo sintetizar» (APCI, 1964: 805). Se presentaba como un primus inter pares, emprendiendo una gestión colegiada del partido (Höbel, 2010: 58). En este escenario, se asistió a una definición del partido con tres direcciones, dos antagónicas propensas a la superación de la forma clásica del partido y una tercera «interesada en gestionar las 'sugestiones' provenientes de las alas para consolidar el papel de aquel partido dentro de las instituciones italianas» (Sorgerà, 2011: 13). Así, en el marco de un delicado equilibrio de fuerzas, desde 1965 las posiciones discordantes se manifestaron claramente, escenificando las diferencias entre la derecha (vinculada a la figura de Amendola), la izquierda (cercana a la figura de Ingrao) y el centro del partido. El secretario Longo asumió el arduo papel de suavizar las demandas de mayor radicalidad o pragmatismo de cada ala, actuando como mediador «entre las diversas almas internas» y favoreciendo la definición del perfil interno del PCI en un escenario tripartido (Sorgerà, 2011: 13-14).

Tanto era así que como en otras ocasiones, ante el peligro de que la fractura se agudizase en el partido, Longo intervino buscando una mediación, suavizando los extremos, marcando la línea de «consenso» a seguir y realizando una síntesis comprensiva de las diferentes posturas. El secretario del PCI contestó al artículo de Amendola en una entrevista publicada en Rinascita, en la que reflexionaba sobre los sucesos franceses ${ }^{17}$. Longo indicaba que, tanto para el PCF como para el PCI, el principal problema era la «conquista de posiciones nuevas, políticas, dentro de las fábricas y en el país». Incidía en la necesidad que el PCI dialogase con el movimiento con el espíritu abierto «sin ninguna presunción» y añadía: «La enseñanza que nos viene de la experiencia francesa es que la lucha democrática puede y debe enriquecerse de nuevas formas de lucha, que no solo a través de los tradicionales métodos de lucha sindical se puede incidir sobre la situación política». Longo subrayaba cómo existían una serie de formas de lucha, «nuevas formas de organización, de poder y de control en el país».

17. «Riflessioni sugli avvenimenti di Francia. Colloquio con Luigi Longo». Rinascita, 24, 1968. 
Pese al buen resultado electoral, el debate sobre la relación con el movimiento estudiantil, siguió vivo. En los Comités del Partido, en los medios (LUnità y Rinascita, sobre todo), se pronunciaban discursos y se publicaban textos acerca de la línea que debía mantenerse con el movimiento estudiantil.

Concluyendo, no hay unanimidad de juicios sobre el papel del partido ante esta complicada situación. Para algunos, «la difícil relación entre el PCI y la contestación estudiantil terminó por resolverse, como para otras fuerzas políticas, en un encuentro fallido» (Santarelli, 1997: 152). Para otros, el PCI había conseguido obtener beneficio en virtud de su estrategia basada en el diálogo con el movimiento estudiantil. Durante 1968, el PCI demostró capacidad de comprender las demandas y las reivindicaciones tanto de los trabajadores como de los estudiantes, deteniendo, al menos en esta fase, el atractivo de los nuevos grupos de extrema izquierda que se erigían baluarte en defensa de la unidad del movimiento obrero y del movimiento estudiantil.

En mayo, el PCI había sido votado por los jóvenes que le reconocían la voluntad y buena predisposición de «acercarse» a los manifestantes. El partido había hecho un esfuerzo para analizar las dinámicas del movimiento, superando las resistencias internas dentro de la parte conservadora del mismo. Respaldado por la «izquierda» del partido, Longo y su círculo más cercano habían promovido una línea política moderada, favoreciendo «una mutación de los equilibrios internos respecto al XI Congreso» (Höbel, 2004: 445). Para evitar una posible hemorragia de militantes y votos, había emprendido un viraje «hacia la izquierda» que se confirmó en ocasión del XII Congreso del PCI (febrero de 1969). En su intervención, el secretario calificó la actitud del partido hacia el movimiento como particularmente positiva a la vez que invitaba a «vincular los movimientos más avanzados y las posiciones más radicales con las más amplias capas del pueblo italiano» (AA.VV., 1969b: 37). Al mismo tiempo seguía privilegiando a la clase obrera como interlocutor principal, prestándole especial atención para comprender su fuerza de movilización antes del estallido del «autunno caldo» de 1969.

No obstante, era evidente que dentro del PCI se pospuso el debate que estaría presente en los setenta entre dos posturas contrapuestas: los partidarios de una renovación de la estrategia y del funcionamiento del partido en un sentido más acorde a las sociedades donde actuaban (eurocomunismo), y aquellos que apostaban por un repliegue identitario, una defensa del socialismo soviético.

La primera postura fue la ganadora. Durante el XIII Congreso del Partido Comunista italiano (marzo de 1972), Enrico Berlinguer fue elegido secretario general del PCI. La decisión de ascender a Berlinguer a tan importante cargo fue consensuada y, por su parte, Longo confesó haberle elegido «por la 
contribución ideológica dada al debate comunista, por su coherencia y su rigor en la defensa de la democracia en el partido y también un poco por su tozudez sarda» (Lajolo, 1975: 116). En sus primeras declaraciones, el nuevo secretario discutió la necesidad de «realizar un giro democrático, que modifique los fines y la calidad del desarrollo económico-social, cambie la posición de las masas trabajadoras en la vida nacional, dé una dirección política al país» (Berlinguer, 1977: 111).

\section{Epílogo y conclusión}

En Italia 1968 fue «el año de los estudiantes» (Rossanda, 1968): tuvo un importante valor simbólico, pero inicialmente desde el punto de vista político su peso fue más reducido. Significó un cambio social importante y el inicio de una nueva etapa en las relaciones entre fuerzas políticas y movimientos sociales. Su indudable relevancia socio-psicológica se debió también al papel de los medios de comunicación que contribuyeron a la difusión del movimiento.

A lo largo de las páginas de este artículo se ha puesto de manifiesto la existencia de un movimiento italiano previo al francés, con el que compartió estrategias y reivindicaciones, pero que se diferenció en varios aspectos debido a las peculiaridades nacionales.

El «largo 68 italiano», el período que va entre finales de los años sesenta e inicio de los setenta se caracterizó por la movilización estudiantil y por el «otoño caldo» de los obreros: fueron momentos que ponían de manifiesto «el rechazo de un sistema por parte de sus herederos designados [...] de hecho certificaron el agotamiento de una agenda política y la apertura de una nueva» (Maier, 1993: 342). Si ya en 1969 se asistió a una lenta desmovilización del movimiento estudiantil (evidente en otoño de este mismo año), de manera inversamente proporcional aumentó la beligerancia del movimiento obrero. Desde finales de 1969, Italia se encaminaba hacia una nueva etapa denominada de tensión o terror, como forma subversiva de intervención en la política (Biscione, 2012: 18). En realidad, un primer «uso de explosivos en la lucha política se observa ya desde marzo de 1968 en coincidencia con el momento más intenso de la protesta estudiantil» (Dondi, 2015).

Pese a demostrar una actitud propositiva y abierta hacia el movimiento estudiantil, al Partido Comunista italiano le costó comprender que se trataba de una protesta política y cultural. Eran jóvenes política-ideológicamente activos, críticos con el sistema. La postura que asumió el partido marcó su porvenir: determinó un giro a la izquierda del grupo dirigente y una puesta en cuestión de la estrategia del partido en aras de una mayor apertura. El PCI intentó 
recoger las demandas de una sociedad en transición aun manteniendo reservas sobre su estrategia. No obstante, fue un momento importante en la historia del partido. De acuerdo con Höbel, la secretaría de Longo constituyo «mucho más que una mera fase de transición» (2010: 607) y el 68 italiano influyó en la dinámica interna del PCI de los años venideros. Ya en los setenta, el PCI estaba a la búsqueda de un camino diferente, una estrategia propia, aunque en parte representase un «salto al vacío».

1968 fue el año en que el PCI empezó a plantear nuevas exigencias para la hegemonía en todos los órdenes de la vida social. A partir de esta fecha, el Partido Comunista italiano intentó recoger la demanda de parte de la sociedad ansiosa de un nuevo comunismo más realista y democrático, dispuesto a una autocrítica sin llegar a ser herético o iconoclasta: una nueva formulación que tuviera en cuenta las peculiaridades del momento y las exigencias ciudadanas (Donofrio, 2018). Con Berlinguer como secretario el PCI se adoptó una nueva táctica definida Compromiso Histórico, un intento de alcanzar, en el conjunto del país y, sobre todo, en el Parlamento, una «unidad» basada en la mayoría del pueblo italiano, que consiguiera agrupar a las tres principales fuerzas políticas de la sociedad -comunistas, católicos y socialistas-. El objetivo era interpretar las necesidades de la sociedad civil italiana.

El pensamiento de Berlinguer, de inspiración gramsciana, tenía el siguiente punto de partida: el papel del Partido Comunista consistía en articular el máximo de fuerzas sociales y políticas para conseguir una transformación progresiva dentro del marco de la Constitución existente. Definiendo la necesidad de un nuevo giro dentro de la democracia, el PCI perseguía la creación de una alternativa democrática. En realidad, la política de alianzas del PCI daba continuidad a las ideas de Togliatti: buscaba la formación de una agregación de fuerzas progresistas y antifascistas por encima de la tradicional visión bipolar de la sociedad; y la realización de un pacto electoral de izquierda, donde el resultado natural habría de ser la hegemonía del partido-vanguardia.

Finalmente, el PCI de Berlinguer con otros partidos comunistas europeos estaba trabajando para adecuar la doctrina marxista y la acción política del partido a la realidad histórica y política de cada país, buscando ofrecer una contribución original al pensamiento marxista. En el escenario internacional, aparecía un sugestivo y fascinante proyecto: el eurocomunismo. 


\section{Fuentes}

ARCHIVO DEL PARTIDO COMUNISTA ITALIANO (APCI), Dirección, microfilm 28 (1964) y 20 (1968).

\section{Bibliografía}

AA.VV. (1969a). Documenti politici dall'XI al XII Congresso. Roma: Editori Riuniti. AA.VV. (1969b). XII Congresso del Partito comunista italiano. Atti e risoluzioni. Roma: Editori Riuniti.

BENCI, Antonio (2009). 'È scoppiata la rivoluzione...'. Il maggio francese e il movimento del Sessantotto in Italia, Storicamente, 5 , n. ${ }^{\circ}$ 11. <http://doi.org/10.1473/ stor53>

BERLINGUER, Enrico (1977). Gobierno de unidad democrática y compromiso histórico: discursos 1969-1976. Madrid: Ayuso.

BISCIONE, Francesco M. (2012). Una modalità della lotta politica in Italia 19691974. En Carlo GHEZZI. Brescia: Piazza della Loggia (17-40). Roma: Ediesse.

CHIARANTE, Giuseppe (1968). La rivolta degli studenti. Roma: Editori Riuniti.

CHIARANTE, Giuseppe (1996). Da Togliatti a D’ Alema. La tradizione dei comunisti italiani e le origini del PDS. Roma-Bari: Laterza.

CHIAROTTO, Francesca (2017). Aspettando il sessantotto. Continuità e fratture nelle culture politiche italiane dal 1956 al 1968. Torino: Accademia University Press.

COCCIA, Benedetto (2008). Quarant'anni dopo: il sessantotto in Italia fra storia, società e cultura. Roma: Apes.

COLANICCHIA, Ingrid; RUSSO SPENA, Giacomo (2018). Una tigre di carta contro gli studenti, MicroMega, 1/2018.

DE LUCA, Francesco (1968). Il PCI e il movimento studentesco, LEuropa, 29.06.1968. DI MAGGIO, Marco (2014). Alla ricerca della terza via al socialismo. Napoli: Edizioni Scientifiche Italiane.

DONDI, Mirco (2015). Leco del boato: storia della strategia della tensione 1965-1974. Roma-Bari: Laterza.

DONOFRIO, Andrea (2018). Érase una vez el eurocomunismo. Las razones de un fracaso. Madrid: Tecnos.

FLORES D'ARCAIS, Paolo (2018). Gli anni dell'incanto libertario. MicroMega, $1 / 2018$.

GINSBORG, Paul (2003). A history of contemporary Italy. Nueva York: Palgrave Macmillan.

GRISPIGNI, Marco (2018). El poder está en la calle. Violencia y movimientos sociopolíticos en el largo 68 italiano. Madrid: La Oveja Roja.

GRAMSCI, Antonio (1975). Quaderni del carcere, vol. III. Torino: Einaudi.

HÖBEL, Alexander (2004). Il PCI di Longo e il '68 studentesco. Studi Storici, 2, 419-459. 
HÖBEL, Alexander (2010). Il Pci di Luigi Longo, 1964-1969. Napoli-Roma: Edizioni scientifiche italiane.

LAJOLO, Davide (1975). Finestre aperte a Botteghe oscure. Milano: Rizzoli.

MAIER, Charles S. (1993). I fondamenti politici del dopoguerra. En Perry ANDERSON et al. (coord.). Storia d'Europa, vol. I: L'Europa oggi (313-372). Torino: Einaudi.

MARZILLO, Massimiliano (2012). Lopposizione bloccata. PCI e centro-sinistra (1960-1968). Soveria Mannelli: Rubbettino.

ROSSANDA, Rossana (1968). Lanno degli studenti. Bari: De Donato.

SANTARELLI, Enzo (1997). Storia critica della repubblica: l'Italia dal 1945 al 1994. Milano: Feltrinelli.

SORGONÀ, Gregorio (2011). La svolta incompiuta. Il gruppo dirigente del Pci tra l'VIII e l’XI congresso (1956-1965). Roma: Aracne.

TOLOMELLI, Marica (2015). L'Italia dei movimenti. Politica e società nella Prima repubblica. Roma: Carocci. 